\title{
Bistability of Contact Angle and Its Role in Achieving Quantum-Thin Self-Assisted GaAs nanowires
}

Wonjong Kim, ${ }^{\dagger}$ Vladimir G. Dubrovskii, ${ }^{\ddagger}$ Jelena Vukajlovic-Plestina, ${ }^{\dagger}$ Gözde Tütüncüoglu, ${ }^{\dagger}$ Luca Francaviglia, ${ }^{\dagger}$ Lucas Güniat, ${ }^{\dagger}$ Heidi Potts, ${ }^{\dagger}$ Martin Friedl, ${ }^{\dagger}$ Jean-Baptiste Leran, ${ }^{\dagger}$ and Anna Fontcuberta i Morral* ${ }^{*} \dagger$ (i)

${ }^{\dagger}$ Laboratory of Semiconductor Materials, Institute of Materials, École Polytechnique Fédérale de Lausanne, 1015 Lausanne, Switzerland

${ }^{\ddagger}$ ITMO University, Kronverkskiy pr. 49, 197101 St. Petersburg, Russia

\section{Supporting Information}

\begin{abstract}
Achieving quantum confinement by bottom-up growth of nanowires has so far been limited to the ability of obtaining stable metal droplets of radii around $10 \mathrm{~nm}$ or less. This is within reach for gold-assisted growth. Because of the necessity to maintain the group III droplets during growth, direct synthesis of quantum sized structures becomes much more challenging for self-assisted III-V nanowires. In this work, we elucidate and solve the challenges that involve the synthesis of galliumassisted quantum-sized GaAs nanowires. We demonstrate the existence of two stable contact angles for the gallium droplet on top of GaAs nanowires. Contact angle around $130^{\circ}$ fosters a continuous increase in the nanowire radius, while $90^{\circ}$ allows for the stable growth of ultrathin tops. The experimental results are fully consistent with our model that explains the observed morphological evolution under the two different scenarios. We provide a generalized theory of self-assisted III-V nanowires that

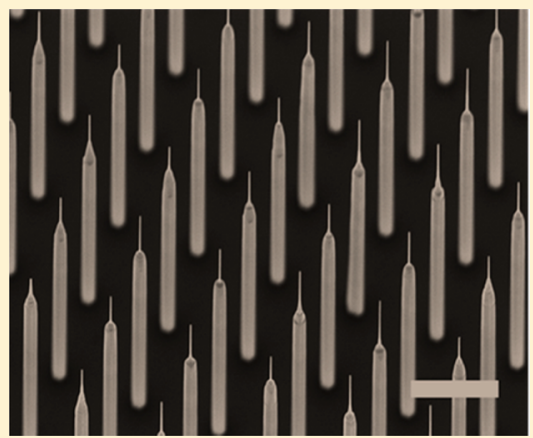
describes simultaneously the droplet shape relaxation and the NW radius evolution. Bistability of the contact angle described here should be the general phenomenon that pertains for any vapor-liquid-solid nanowires and significantly refines our picture of how nanowires grow. Overall, our results suggest a new path for obtaining ultrathin one-dimensional III-V nanostructures for studying lateral confinement of carriers.

KEYWORDS: III-V semiconductors on silicon, nanowires, nanoneedles, regular arrays, self-assisted growth, droplet size engineering, crystal structure, growth modeling
\end{abstract}

$\mathrm{F}$ undamental physical properties and applications of semiconductor nanowires (NWs) may benefit from quantum confinement due to size-dependent modulation of the density of states as well as modification of the photon energy for the allowed optical transitions. ${ }^{1}$ In GaAs, quantum confinement occurs for a size below $25 \mathrm{~nm}^{2,3}$ In addition to quantum confinement, the optical properties can be engineered by modifying the NW shape. For example, progressively tapered nanowires have exhibited adiabatic outcoupling in the emission of quantum dots, thereby enhancing their brightness. ${ }^{4}$ A similar design may improve the light extraction or absorption in different optoelectronic devices including light emitting diodes, solar cells, and optical biosensors. $^{5-11}$ Quantum confinement in the core of radial NW heterostructures allows for the fabrication of high quality NW-based quantum dots, which are a perfect platform for delicate quantum transport experiments. ${ }^{12,13}$

It is admittedly challenging to obtain very thin (quantumconfined) III-V NWs directly by the vapor-liquid-solid (VLS) growth technique. There are several reasons for that (see ref 14 for a review), including the Gibbs-Thomson effect of elevation of chemical potential due to the curvature of the droplet surface and difficulties in obtaining very small droplets on the substrate surface. Thin tips of VLS GaAs NWs with a stable radius down to $5 \mathrm{~nm}$ have previously been grown by hydride vapor phase epitaxy ${ }^{15,16}$ (a technique that involves very large material inputs and consequently high supersaturations in the liquid phase) but with low density and random position. III-V nanoneedles (NNs) with atomically sharp tip ( $\sim 1 \mathrm{~nm}$ in radius) have also been fabricated in the catalyst-free approach in ref 17 . Gallium-assisted growth of GaAs NWs is of particular interest as a platform for monolithic integration of III-V photonic nanostructures with silicon electronics. It has been demonstrated that ordered arrays of GaAs NWs with high yields can be obtained by the galliumassisted molecular beam epitaxy (MBE) growth on patterned $\mathrm{SiO}_{x} / \mathrm{Si}(111)$ substrates. ${ }^{18-20}$ In this specific case, achieving small NW radii is even more challenging because galliumassisted GaAs NWs are usually grown under effectively gallium-rich conditions to maintain the gallium droplets on

Received: July 22, 2017

Revised: November 12, 2017

Published: December 19, 2017 


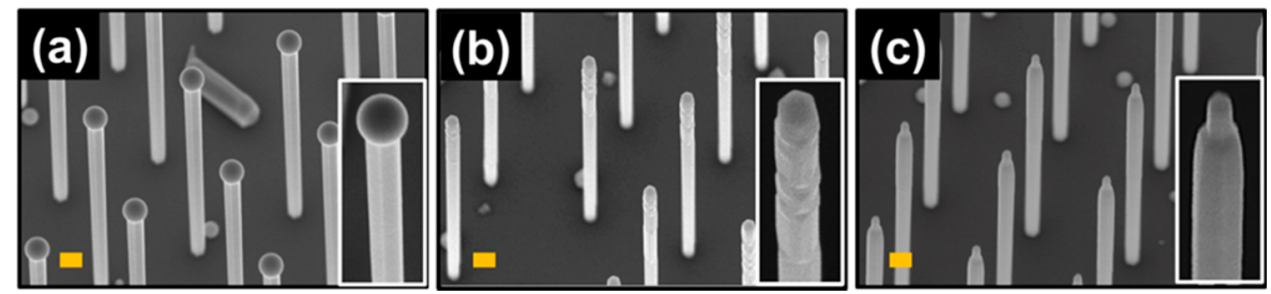

Figure 1. Twenty degree tilted SEM images showing the regular GaAs NWs with an array pitch of $1000 \mathrm{~nm}$ : (a) reference NWs having large gallium droplets on tops, transitioning to the NWs with tapered tops and without any droplets after 20 min under the As $\mathrm{B}_{4} \mathrm{BEP}$ of $(\mathrm{b}) 7.2 \times 10^{-7}$ Torr and (c) $2.0 \times 10^{-6}$ Torr. The scale bars correspond to $200 \mathrm{~nm}$. The inserts show zoomed images of standard NWs and NWs without droplets with flat tops.

top of NWs. In self-assisted VLS growth of GaAs NWs, the droplet serves as a nonstationary reservoir of gallium. ${ }^{21-25}$ Hence, the amount of gallium changes during growth and the droplet is able to either shrink under arsenic-rich conditions, swell under gallium-rich conditions, or self-equilibrate to a certain stable size under a balanced V/III flux ratio. ${ }^{21-23}$ Gallium-rich conditions at the beginning of growth necessarily lead to the droplet swelling, which is why the NW radius usually increases with respect to the initial droplet size as, for example, in refs 21, 22, and 26.

Herein, we propose a new strategy for the fabrication of ultrathin self-assisted GaAs NNs on top of GaAs NWs grown on silicon. To obtain spatially homogeneous ultrathin structures in a highly reproducible manner, GaAs NWs are grown by $\mathrm{MBE}$ in regular arrays on patterned $\mathrm{SiO}_{x} / \mathrm{Si}(111)$ substrates. The homogeneous position and spacing allow for achieving uniform shape distribution over the substrate surface because the growth mechanisms of NWs are known to depend on the interwire spacing. ${ }^{27,28}$ The initial growth step is performed under gallium-rich conditions to achieve selfassisted VLS growth of regular GaAs NW stems by the standard procedure described in detail in ref 26. The nominal size of the holes in the oxide layer corresponds to $45 \mathrm{~nm}$. The final hole size is increased by approximately $10 \mathrm{~nm}$ due to the substrate preparation step that includes a short HF dip process to ensures oxide-free surface in the holes. The pitch of the square array of holes is kept in the range of $800-1000 \mathrm{~nm}$. The NW growth is initiated by first depositing gallium, which is collected in predefined holes in the oxide layer. Then the arsenic flux is increased to a beam equivalent pressure (BEP) of $\mathrm{As}_{4}$ of $2 \times 10^{-6}$ Torr and the NWs are grown at a surface temperature of $635{ }^{\circ} \mathrm{C}$ up to a total length of around $4 \mu \mathrm{m}$. Figure 1a shows the scanning electron microscopy (SEM) image of the standard GaAs NWs obtained after $60 \mathrm{~min}$ of growth that have large gallium droplets on their tips, with an average contact angle of $130^{\circ}$. Such large droplets are commonly observed in most gallium-assisted GaAs NWs. ${ }^{18,26,29,30}$

If the gallium supply is turned off and the NWs are left under an arsenic flux, the droplets are fully consumed, as demonstrated in Figure 1 after the exposition to the arsenic fluxes corresponding to the BEPs of $7.2 \times 10^{-7}$ Torr in Figure $1 \mathrm{~b}$ and $2.0 \times 10^{-6}$ Torr in Figure 1c. This is not surprising because all the remaining gallium should crystallize with the available arsenic producing some additional GaAs on the NW tips, as in ref 21 . The degree of tapering at the NW tops is larger for higher arsenic flux. Similarly to ref 21 , the NW tops are completely flat, meaning that the droplets are consumed at a higher rate compared to the rate of NW tapering. At some stage, the droplets become smaller than the NW top facet (otherwise, the tops would have a needle-like shape following the gradually diminishing droplet radius).

We now try to engineer the shape of the NW tops by abruptly increasing the As ${ }_{4}$ BEP under the same gallium flux as in the standard growth step. The idea of this procedure is to shrink the gallium droplets rather than fully consume them, and then try to maintain a small droplet size to produce sharp NNs with a uniform radius on top of NWs. Figure 2a illustrates schematic morphology evolution of NWs. Increasing the $\mathrm{As}_{4}$ BEP to $8.9 \times 10^{-6}$ Torr for 2 min after the standard growth step (Figure $2 b$ ) yields the structures shown in Figure $2 c$. Even though the gallium flux is still on, the droplets have decreased to a radius below that of the NWs. By keeping these conditions for 3 min longer, we observe the formation of conical-like NN tops, as shown in Figure 2d. The top radius of conical-like NNs can be decreased to a very small size, about $5 \mathrm{~nm}$ in the thinnest NNs seen in the figure. We expect the gallium droplets may be fully consumed in a certain stage of this process. After that, the VLS growth should stop due to absence of gallium and NNs cannot grow any longer. So far, we have obtained NNs up to $\sim 2 \mu \mathrm{m}$ in length with that technique.

If, instead of maintaining the arsenic BEP at a constant value of $8.9 \times 10^{-6}$ Torr in the droplet shrinking stage, we decrease it down to $5.5 \times 10^{-6}$ Torr from the step shown in Figure $2 \mathrm{c}$, the morphology changes dramatically. Figure $2 \mathrm{e}$ shows the representative SEM image of the structures obtained after 20 min of growth at $5.5 \times 10^{-6}$ Torr. It is clearly seen that introducing the third growth step with a lower arsenic flux after shrinking the droplets results in the formation of thin NNs that first taper and then acquire a uniform radius of approximately $10 \mathrm{~nm}$. This stationary radius stays constant along $\sim 1 \mu \mathrm{m}$ of the NN length. Figure $2 \mathrm{f}$ and $g$ show lower magnification SEM images of the NNs obtained by the same three-step procedure in arrays with $1000 \mathrm{~nm}$ pitch, further illustrating the achieved high degree of homogeneity in their size and shape. It is possible to grow the NNs even longer. Figure $2 \mathrm{~h}$ shows the SEM of a $5 \mu \mathrm{m}$ long NN achieved by the three-step method. To summarize this part, three-step growth allows us to obtain regular quantum-thin NNs on top of NWs by tuning the arsenic flux only, which has never been achieved before to our knowledge.

To investigate quantum size effects in our structures, we have tested the phonon confinement properties by the Raman spectroscopy. Room temperature Raman spectra of the GaAs NW stem and the NN top obtained in the backscattering geometry and by exciting with a laser at $785 \mathrm{~nm}$ are shown in Figure $2 \mathrm{i}$. The spectra originating from the GaAs stems are composed of two peaks, the TO and LO modes at about 267.5 and $285.2 \mathrm{~cm}^{-1}$, respectively, in agreement with what is found in bulk GaAs. ${ }^{31}$ The Raman spectra of the NNs systematically 

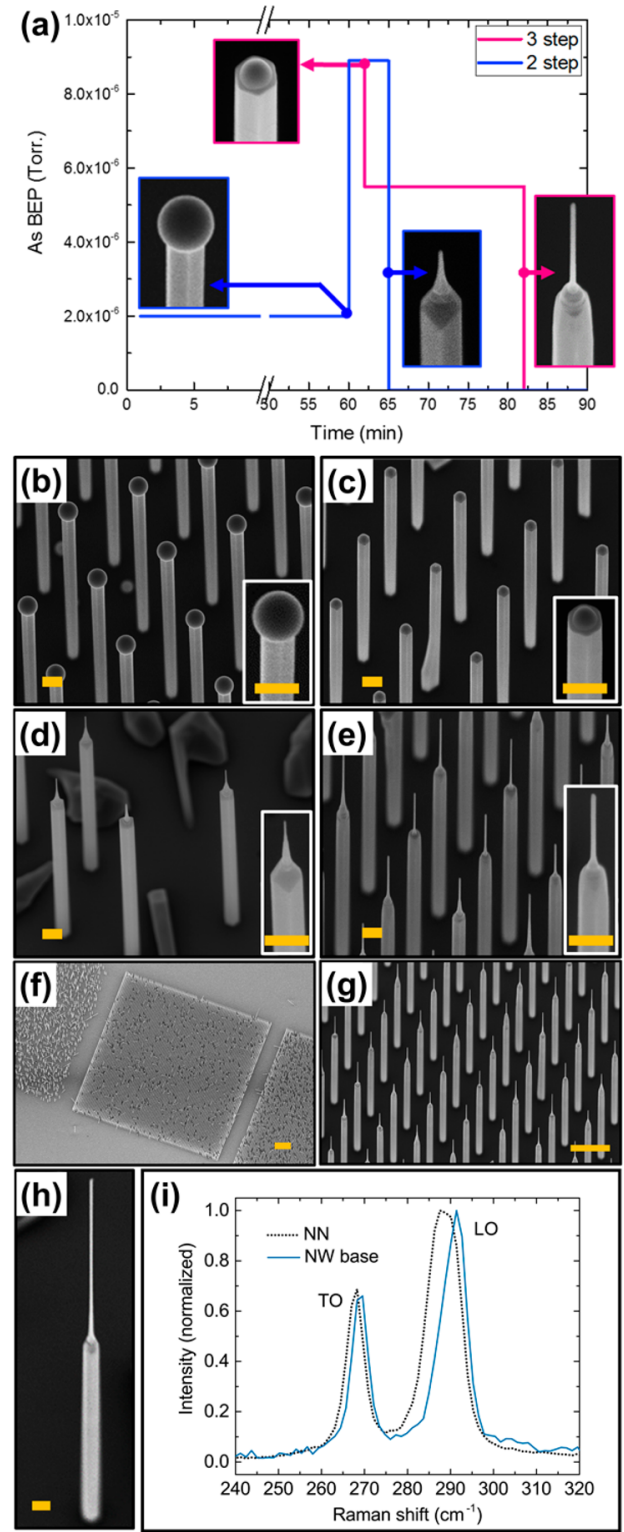

Figure 2. (a) Schematic morphology evolution of NWs and $20^{\circ}$ tilted SEM images of $800 \mathrm{~nm}$ pitch arrays. (b) Standard GaAs NWs growth under gallium-rich conditions at an $\mathrm{As}_{4} \mathrm{BEP}$ of $2.0 \times 10^{-6}$ Torr. (c) Abrupt droplet shrinkage by increasing the $\mathrm{As}_{4}$ BEP to $8.9 \times 10^{-6}$ Torr for 2 min. (d) Further evolution of the tip under the same $\mathrm{As}_{4}$ BEP as in panel c, the tips are conically tapered and the droplets are finally lost. (e) Straight, $\sim 10 \mathrm{~nm}$ radius NNs on top of NWs obtained via the three-step procedure by decreasing the $\mathrm{As}_{4} \mathrm{BEP}$ to $5.5 \times 10^{-6}$ Torr after the step shown in panel $\mathrm{c}$ and growing the NNs for $20 \mathrm{~min}$. The scale bar indicates $200 \mathrm{~nm}$. The inserts in panels $\mathrm{d}$ and e show the tapered tip without any droplet and straight $\mathrm{NN}$ with the droplet for higher and lower $\mathrm{As}_{4} \mathrm{BEP}$, respectively. Regular array of the GaAs NN-on-NW structure with a spacing of $1000 \mathrm{~nm}$. The scale bar indicates $10 \mu \mathrm{m}$ in panel $\mathrm{f}$ and $1 \mu \mathrm{m}$ in panel g. (f) and (g) Lower magnification SEM images of regular NNs on top of NWs. (h) Extended NN tip obtained after 40 min growth via the three-step procedure. The scale bar indicates $200 \mathrm{~nm}$. (i) Raman spectra of the NW stem and the NN obtained in the backscattering geometry at room temperature.

(see SI6) exhibit the two peaks with a clear down-shift of about $2.5 \mathrm{~cm}^{-1}$ for the LO modes and a broadening with respect to the Raman spectra of the NW stems. The downshift and broadening are in clear agreement with phonon quantum- confinement effects in the NN and further illustrate their added functionality, ${ }^{32-34}$ which can be used in many applications discussed above. A modification in the nature of phonons in the NNs could be used, for example, in thermoelectric applications. ${ }^{35}$

It is noteworthy that the gallium droplets on top of the standard NWs and ultrathin NNs exhibit very different shapes; while the first ones are large (with a typical contact angle of $\sim 130^{\circ}$ as shown in Figure $2 \mathrm{~b}$ ), the second are much smaller, with an average contact angle decreasing down to $\sim 90-95^{\circ}$ (as shown in Figure 2c. The droplet that shrinks smaller than the NW top facet in Figure $2 \mathrm{c}$ also exhibits a contact angle around $90^{\circ}$. The effect of droplet contact angle being smaller for higher V/III ratios is well-known for gold-catalyzed III-V NWs. ${ }^{36,37}$ This is explained simply by the fact that the droplet contains more gallium under gallium-rich and less gallium under arsenic-rich conditions and the contact angle changes accordingly. However, in gallium-assisted growth, the droplet is almost pure gallium ${ }^{30}$ and hence its shape change with the arsenic flux is less clear and requires special treatment.

We now address the evolution of the crystal phase along the NW/NN length. Figure 3 presents the transmission electron microscopy (TEM) micrographs of a representative GaAs NW with NN. The whole structure was imaged along the $[1-10]$ and $[11-20]$ axis zone for the zinc-blende (ZB) and wurtzite (WZ) phases, respectively. Figure 3 a shows a low magnification overview of the full-length NW, showing a stem with a homogeneous radius of $75 \mathrm{~nm}$, followed by a progressive radius reduction until stabilization at $10 \mathrm{~nm}$. Figure $3 \mathrm{~b}$ is a zoomed-in TEM image of the NN. High-resolution (HR) TEM images from different parts of the NW and NN are reported in Figure $3 c-e, g, i, k, m$, and o. Selective area electron diffraction (SAED) patterns are given in Figure $3 f, h, j$, $1, \mathrm{n}$, and $\mathrm{p}$. The stem grown under gallium-rich conditions appears pure $\mathrm{ZB}$. The ZB crystal phase obtained here is not different from most gallium-assisted GaAs NWs described in the literature ${ }^{18-20,26,23,38,39}$ (although some WZ segments have also been reported $\left.{ }^{40}\right)$. Predominant ZB phase of galliumassisted GaAs NWs has previously been explained by a low energy of liquid gallium ${ }^{38}$ that suppresses the triple phase line nucleation $^{41}$ (the necessary condition for the WZ phase formation) on surface energetic grounds. More recently, it was shown that some NWs may exhibit a truncated facet at the growth interface and that such truncated geometry occurs for larger contact angles of the droplet. ${ }^{38}$ If the truncated geometry is present all the time, the islands cannot nucleate at the triple phase line and hence the crystal phase should be $\mathrm{ZB}^{36}$

Interestingly, the WZ phase appears in the transition from the NW to the NN geometry and eventually becomes the predominant crystal phase in thin NNs. This is consistent with the result of ref 25, where the arsenic flux modulation also led to the appearance of the WZ phase in thinner GaAs NWs. At about two-thirds of the NW height from the bottom, a transition region is typically observed, shown in Figure $3 \mathrm{k}$ and 1. After this, moving toward the $\mathrm{NN}$, long segments of both $\mathrm{ZB}$ (Figure $3 \mathrm{i}, \mathrm{j}$ ) and WZ (Figure $3 \mathrm{~g}, \mathrm{~h}$ ) coexist. Figure $3 \mathrm{e}$ and $\mathrm{f}$ are taken at the onset of the NW shrinkage into the NN. Although with several defects, the crystal phase of this section is mostly WZ. From here, the WZ phase begins to dominate, even though the $\mathrm{ZB}$ insertions are visible until the very tip of the $\mathrm{NN}$. We also note that the gallium droplet is still present at the $\mathrm{NN}$ tip and its contact angle is close to $90^{\circ}$. The ZB-to-WZ 

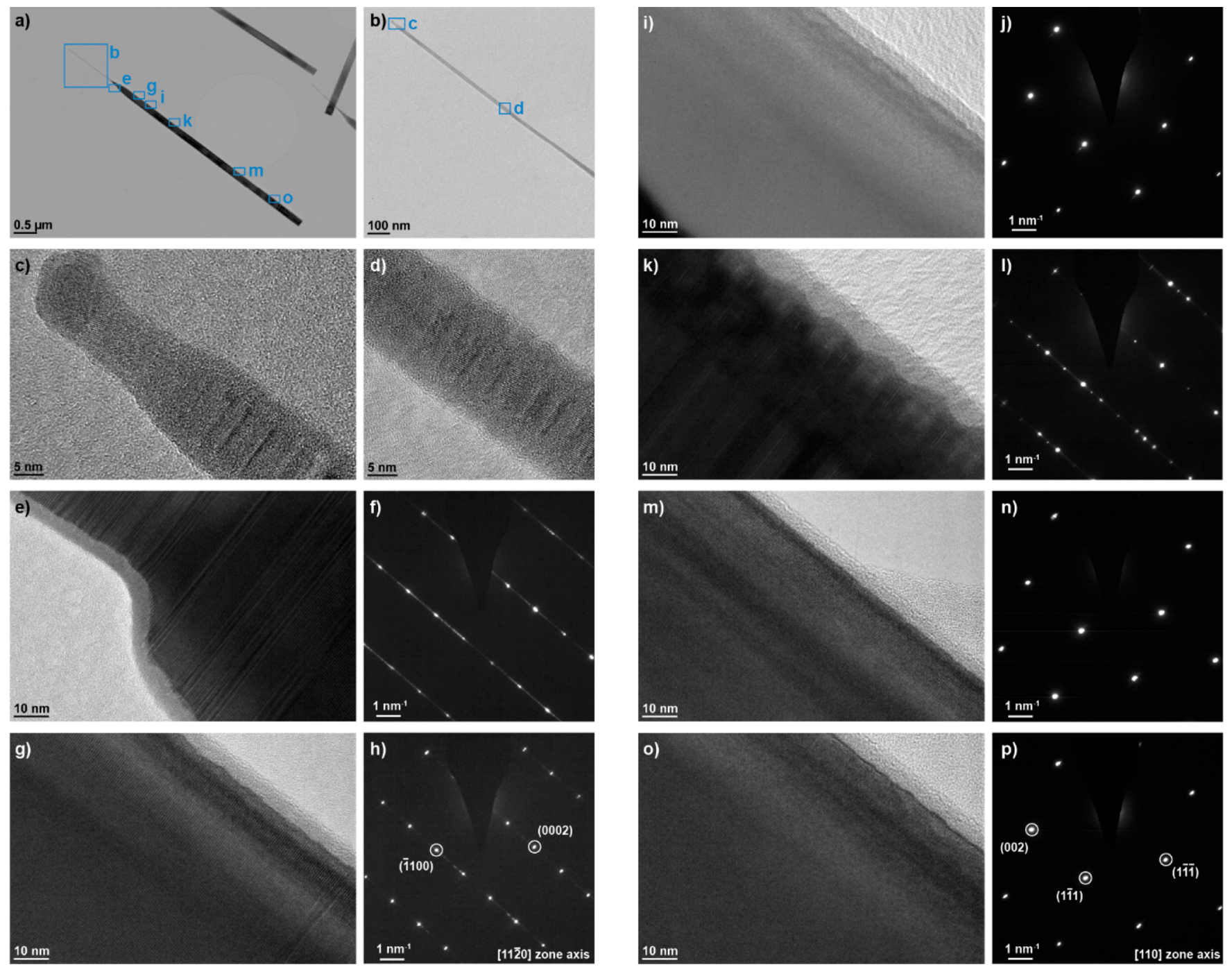

Figure 3. TEM analysis of the crystal phase along the NW and NN. (a, b) Overview of the low magnification TEM micrograph of the entire structure; (c-e, g, i, k, m, o) HR TEM images of different NW sections highlighted in panels a and b; (f, h, j, l, n, p) SAED patterns showing predominantly WZ close to the NN (in panel h) and ZB while moving away from the tip (in panels $j, 1, n$, and $p$ ).

transition occurring as the standard NW shrinks to the thin $\mathrm{NN}$ should be associated with either smaller radius of the NN or smaller contact angle of the droplet catalyzing its growth, similarly to the earlier observations of the WZ phase formation at the very tops of gallium-assisted GaAs NWs. ${ }^{21,38}$

To summarize the experimental results, we were able to shrink the droplet size by abruptly increasing the arsenic flux and then to maintain the stable droplet at the NW top in the three-step MBE procedure with the arsenic flux increasing in the second step and slightly decreasing in the third step. These manipulations produce highly regular straight NNs on top of NWs, with a uniform radius of about $10 \mathrm{~nm}$ over $1 \mu \mathrm{m}$ length or even longer. The crystal phase changes from pure ZB in the NW stems with large contact angle droplets to very predominantly $\mathrm{WZ}$ in the $\mathrm{NNs}$ with small contact angle droplets. Engineering of the morphology of gallium-assisted GaAs NWs by the arsenic flux ${ }^{21}$ as well as the crystal phase trend versus the contact angle in the case of gold-catalyzed III-V NWs ${ }^{36}$ is in principle well-known. The new findings presented here are in demonstration of: (i) the existence of two stable contact angles for self-assisted VLS growth of relatively thick GaAs NWs $\left(\sim 130^{\circ}\right)$ and thin GaAs NNs $\left(\sim 90^{\circ}\right)$, respectively, and (ii) the sharp and well-controlled transition from NWs to NNs having a stable radius of $10 \mathrm{~nm}$, associated with the corresponding change of the crystal phase from pure ZB to mostly WZ. Consequently, below we present theoretical considerations explaining these new results and discuss how they can be extrapolated to other material systems.

We start by providing understanding of the droplet and NW shape evolution under different scenarios. This involves the role of the contact angle as a function of tapering and for configurations in which the droplet is or not wetting the NW side facets, referred to as wetting or nonwetting facets in the following. According to the recent analysis, ${ }^{42}$ in the mononuclear VLS growth regime whereby one monolayer of semiconductor material is instantaneously removed from the catalyst droplet, ${ }^{14,30,42-44}$ the difference of surface energies of forming truncated nonwetted facets $\left(G_{\theta}^{n-w}\right)$ versus vertical nonwetted facet $\left(G_{0}^{n-w}\right)$ is given by

$$
G_{\theta}^{n-w}-G_{0}^{n-w}=\frac{\gamma_{\theta V}}{\cos \theta}-\gamma_{0 V}-\left(\gamma_{S L}+\gamma_{L V} \cos \beta\right) \tan \theta
$$


(a)

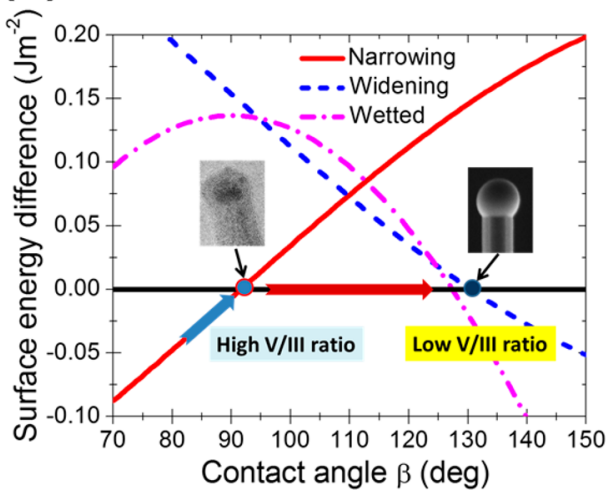

(b)

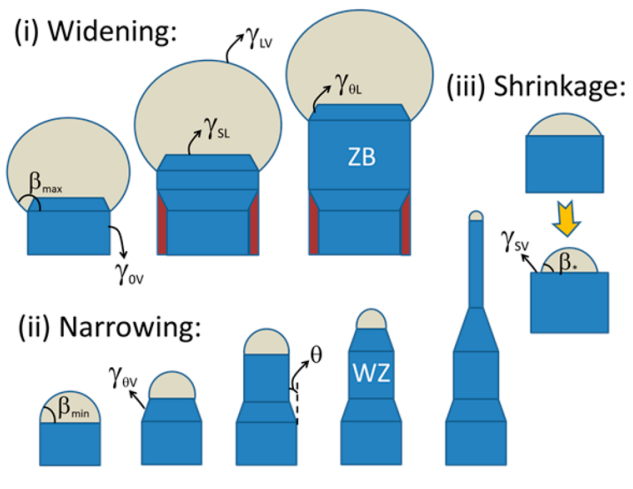

Figure 4. Surface energetics and the overall growth picture. (a) Surface energy curves for narrowing nonwetted $\left(\theta=19.5^{\circ}\right.$, red solid line), widening nonwetted $\left(\theta=-19.5^{\circ}\right.$, blue dashed line) and narrowing wetted $\left(\theta=19.5^{\circ}\right.$, magenta dash-dotted line) facets. Zero level corresponds to the surface energy of vertical nonwetted facets. The two stable contact angles $\beta_{\min }$ and $\beta_{\max }$ approximately equal $90^{\circ}$ and $130^{\circ}$ for our model parameters, corresponding to the experimentally observed values. At low V/III flux ratios, the droplet volume increases by increasing the contact angle until $\beta_{\max }$. After that, the stable contact angle is maintained by introducing widening facets and the droplet volume further increases by increasing the top radius of the NW. Above $\sim 127^{\circ}$, the growth front becomes truncated. At high V/III ratios, the minimum contact angle $\beta_{\text {min }}$ is reached by inward tapering at the beginning, and then maintained by introducing narrowing facets, which is why the NW top radius shrinks. (b) Growth picture under gallium-rich conditions (widening at $\beta_{\max }$ ), with introduction of widening facets and increasing the NW radius first at the top and then along the entire length by step flow; NW narrowing at $\beta_{\min }$ by introducing narrowing facets and shrinking the top radius until the droplet volume stabilizes. This asymptotic stage corresponds to straight NN on top of NW. When the arsenic flux is very high, the contact angle may decrease below the equilibrium Young's angle $\beta_{*}$ and then the droplet should shrink smaller than the top facet to resume the equilibrium shape (the droplet shrinkage). The crystal phase of the NW stems is ZB because their growth interface is wetted truncated. Predominantly WZ crystal phase of the NN tops is explained by the planar growth front at $\beta_{\min }$, which allows for the triple phase line nucleation.

Here, $\gamma_{\theta V}$ are the solid-vapor surface energies of the tapered NW side facets inclined by the angles $\theta$ with respect to the vertical, $\gamma_{O V}$ is the solid-vapor surface energy of the vertical facet, $\gamma_{S L}$ is the solid-liquid surface energy of the NW top facet, $\gamma_{L V}$ is the liquid-vapor surface energy, and $\beta$ is the contact angle of the droplet. On the other hand, the difference of surface energies of forming truncated wetted facet $\left(G_{\theta}^{w}\right)$ versus vertical nonwetted facet equals ${ }^{42}$

$$
G_{\theta}^{w}-G_{0}^{n-w}=\frac{\gamma_{\theta L}}{\cos \theta}-\gamma_{0 V}-\gamma_{S L} \tan \theta+\gamma_{L V} \sin \beta
$$

with $\gamma_{\theta L}$ as the solid-liquid surface energy of the truncated wetted facet. The surface energies and the geometry used in the model are illustrated in Figure 4.

It has often been assumed ${ }^{14,21,22,30,42}$ that, for a given material combination, the contact angle of the droplet is fixed by the surface energetics and hence is maintained by changing the NW top radius under varying V/III flux ratio. This property would have been particularly well justified for galliumcatalyzed GaAs NWs because in this case the droplet is almost pure gallium regardless of the group $\mathrm{V}$ flux, and is seated on top of pure GaAs. However, our experimental results clearly show the occurrence of the two stable contact angles in the stages of the NW radial growth and shrinking, suggesting that the real growth picture is more complex. We note that the necessary refinement of the growth theory can be obtained simply by analyzing more carefully eq 1 . Indeed, the Nebol'sinShchetinin value of the stable contact angle in the standard nonwetting VLS mode, $\cos \beta_{s}=-\gamma_{S L} / \gamma_{L V}$ (ref 45) follows from eq 1 only if $\gamma_{\theta L} / \cos \theta=\gamma_{0 V}$, that is, when the surface energy of the NW sidewalls is insensitive to their orientation. In this case, the only stable configuration of the droplet is present with the contact angle $\beta_{s}$ because any increase or decrease of the contact angle will immediately be compensated by introducing widening $(\theta<0)$ or narrowing $(\theta>0)$ truncated facet, respectively.

We speculate, however, that this simple situation is unlikely because the initial (110) sidewall surface in the case of cubic (ZB) NWs should be stable against faceting and hence $\gamma_{\theta L} / \cos$ $\theta>\gamma_{O V}$. When this condition is fulfilled, the surface energies of narrowing and widening facets cross with the one of vertical facet at two different contact angles, as shown in Figure 4a. The left crossing point corresponds to the minimum stable contact angle $\beta_{\min }$ for the VLS growth with vertical facets; any decrease of $\beta$ below $\beta_{\min }$ (due to a decreasing droplet volume) is compensated by introducing narrowing facets and the top NW radius shrinks. The right crossing point corresponds to the maximum stable contact angle $\beta_{\max }$ for vertical growth, whose value is maintained by introducing widening facets as the droplet swells and the top NW radius increases. Vertical growth with a time-independent top radius should be possible for any contact angle between $\beta_{\min }$ and $\beta_{\max }$ because the droplet is allowed to change its contact angle without introducing any truncation of the growth interface. This picture should hold for any $<111>$-oriented NWs whose sidewall facets are stable against faceting, not necessarily selfcatalyzed III-V NWs. Of course, the particular values of the stable contact angles $\beta_{\min }$ and $\beta_{\max }$ are defined by the surface energies entering eq 1 and hence will change depending on the NW material and the catalyst metal.

The curve given by eq 2 intercepts with zero at a contact angle corresponding to the energetically preferred introduction of wetted truncated facet at the growth front, which can only grow to a finite size above the nonwetted NW base. ${ }^{46}$ If this angle is smaller than $\beta_{\max }$ but larger than $\beta_{\min }$ as shown in Figure $4 \mathrm{a}$, the transition between the stable contact angles $\beta_{\text {min }}$ and $\beta_{\max }$ is associated with the corresponding change in geometry of the growth interface, from nonwetted vertical at $\beta_{\min }$ to wetted truncated at $\beta_{\max }$. According to the current 
view, ${ }^{36,42,46}$ such growth picture fully explains the observed crystal phase trend of our GaAs NWs. For vertical NWs at $\beta_{\text {min }}$ nucleation of two-dimensional islands occurs at the triple phase line ${ }^{41}$ and the crystal phase is predominantly WZ, with some insertions of $\mathrm{ZB}$ layers. For truncated growth interface at $\beta_{\text {max }}$ islands cannot nucleate at the triple phase line ${ }^{46}$ and the crystal phase is pure $\mathrm{ZB}$.

The dependences of the surface energy difference on the contact angle given in Figure 4a, yielding our experimentally observed stable contact angles around $90^{\circ}$ and $130^{\circ}$, were obtained by using $\gamma_{L V}=0.67 \mathrm{~J} / \mathrm{m}^{2}$ for pure gallium liquid at the growth temperature, ${ }^{38} \gamma_{0 V}=0.81 \mathrm{~J} / \mathrm{m}^{2}$ for the lowest energy (110) side facets of ZB GaAs NWs (ref 47), $\gamma_{S L}=\gamma_{\theta L}=0.40 \mathrm{~J} /$ $\mathrm{m}^{2}$ (ref 48), and $\theta= \pm 19.5^{\circ}$ as for (111)A and (111)B facets of ZB III-V NWs. ${ }^{{ }^{41}}$ The unknown surface energies of truncated facets were adjusted to get the measured values of $\beta_{\min }=90^{\circ}$ and $\beta_{\max }=130^{\circ}$, which can be ensured, for example, by choosing $\gamma_{\theta V} / \cos \theta-\gamma_{0 V}=0.135 \mathrm{~J} / \mathrm{m}^{2}$ and $0.012 \mathrm{~J} / \mathrm{m}^{2}$ for narrowing and widening nonwetted facets, respectively. With these model parameters, the overall growth picture is shown in Figure 4b. Scenarios "(i) Widening" and "(ii) Narrowing" schematize the VLS growths with the time-independent contact angles and the NW radius increasing or shrinking depending on the V/III flux ratio (low or high). When the top NW radius increases, step flow growth starting from the outward tapered facets helps to preserve the uniform NW radius from base to top, as in ref 49 . Stabilization of the NW radius should be due to the droplet volume reaching its stationary value for a given $\mathrm{V} / \mathrm{III}$ ratio, leading to a $\mathrm{NN}$ with constant radius. Finally, the droplet is stable against random horizontal shift smaller than the top facet only when the contact angle exceeds the equilibrium Young's angle $\beta_{*}$ given by $\cos \beta_{*}=\left(\gamma_{S V}-\gamma_{S L}\right) / \gamma_{L V}$, with $\gamma_{S V}$ as the solid-vapor surface energy of the top facet (ref 39). The Young's angle equals approximately $70^{\circ}$ at $\gamma_{S V}=0.64 \mathrm{~J} / \mathrm{m}^{2}$ (the surface energy of (111) top plane should be about $20 \%$ lower than that of (110) plane according to ref 48). For high enough arsenic flux, the contact angle may decrease faster than the inward tapered facets are introduced and then shrink smaller than the top facet to resume the equilibrium droplet shape, as illustrated by scenario "(iii) Shrinkage" in Figure 4b. This scenario explains why the top facets are flat after the complete droplet consumption (Figure 1b,c) and why the droplets shrink smaller than the NW in Figure $2 c$. Increasing the contact angle above $\beta_{*}$ and finally acquiring the stable angle $\beta_{\text {min }}$ is most probably due to the initial tapering of the NNs restricted by the energetically preferred narrowing nonwetted facets, as in ref 39.

To quantify the observed shapes of the NN-on-NW structures, we write down the two kinetic equations for the axial NW growth rate and the time evolution of the total number of gallium atoms in the droplet:

$$
\begin{aligned}
& \frac{\mathrm{d} L}{\mathrm{~d} t}=\varphi_{5} \Omega_{35} I_{5}(1+a) \\
& \frac{\mathrm{d} N_{3}}{\mathrm{~d} t}=\pi R^{2}\left[\varphi_{3} I_{3}-\varphi_{5} I_{5}(1+a)+\frac{2 \Lambda_{3}}{\pi R} I_{3}\right]
\end{aligned}
$$

The elongation rate $d L / d t$ is assumed proportional to the arsenic atomic influx $I_{5}$, as in refs 21 and 22, and includes the fraction of re-emitted species $a I$ (refs 30 and 50), with a certain factor $a$, which depends on the contact angle $\beta$. The $\Omega_{35}=$ $0.0452 \mathrm{~nm}^{3}$ is the elementary volume of solid GaAs and $\varphi_{5}$ is the geometrical factor of MBE growth that depends on the arsenic beam angle $\alpha_{5}$ and the droplet contact angle $\beta$ as given in ref 51 . Theoretical justification of eq 3 can be found in refs 30 and 52, and experimental verifications in refs 29, 30, and 50. In simple terms, the elongation rate scales linearly with the arsenic flux because the droplet is a reservoir of gallium and highly volatile arsenic species are not known to diffuse on the NW sidewalls. According to eq 4, the number of gallium atoms in the droplet $N_{3}$ changes with time due to the gallium to arsenic influx imbalance ${ }^{21}$ because the arsenic influx equals the gallium sink due to NW growth. The total influx of gallium contains the direct impingement rate $\varphi_{3} I_{3}$, with $I_{3}$ as the vapor flux of gallium and $\varphi_{3}$ as the geometrical factor for gallium that depends on the gallium beam angle $\alpha_{3}$ and the contact angle $\beta$. Additionally, there is a diffusion collection of gallium adatoms from the top NW part with the effective collection length $\Lambda_{3}$. The last contribution in eq 4 is inversely proportional to $R$ if $\Lambda_{3}$ is treated as radius-independent. ${ }^{53}$

In gallium-assisted VLS growth, the droplet overwhelmingly consists of gallium. ${ }^{21-25,30,52}$ Hence, we can write $N_{3} \Omega_{3} \cong$ $\left(\pi R^{3} / 3\right) f(\beta)$, with $\Omega_{3}=0.02 \mathrm{~nm}^{3}$ as the elementary volume of liquid gallium and $f(\beta)=(1-\cos \beta)(2+\cos \beta) /[(1+\cos \beta)$ $\sin \beta]$ as the geometrical function relating the volume of spherical cap droplet to the cube of its base $R$, which equals the top NW radius. In our MBE system, the arsenic and gallium beams are $45^{\circ}$ inclined to the substrate normal $\left(\alpha_{3}=\alpha_{5}=\alpha=\right.$ $\left.45^{\circ}\right)$ and thus the geometrical factors for arsenic and gallium equal each other and depend only on the contact angle $\left[\varphi_{3}=\right.$ $\left.\varphi_{5}=\varphi(\beta)\right]$. Eqs 3 and 4 are then reduced to

$$
\frac{\mathrm{d}}{\mathrm{d} L}\left[\frac{R^{3}}{3} f(\beta)\right]=\frac{\Omega_{3}}{\Omega_{35}} R^{2}\left[1-\frac{I_{3}}{I_{5}(1+a)}+\frac{2 \Lambda_{3}}{\pi R \varphi(\beta)} \frac{I_{3}}{I_{5}(1+a)}\right]
$$

In principle, this equation allows one to treat the shape evolution in terms of both the NW top radius and the contact angle. ${ }^{23}$ According to our analysis, however, the droplet should first acquire one of the two stable contact angles $\left(\beta_{\min }\right.$ at high $\mathrm{V} / \mathrm{III}$ ratios and $\beta_{\max }$ at low $\mathrm{V} / \mathrm{III}$ ratios) before the NW radius starts to change. Therefore, we can take a time-independent value of $\beta$ in the stage of radius relaxation. ${ }^{21,22}$ By integrating eq 5 at $\beta=$ const with the initial condition $R\left(L=L_{0}\right)=R_{0}$, where the NW length $L_{0}$ and radius $R_{0}$ relate to the beginning of each growth step, after some simple manipulations we get explicitly the relationship between the NW length and the tip radius in the form

$$
L=L_{0}+\frac{F_{53}}{\varepsilon\left(F_{53}-1\right)}\left[R_{0}-R+R_{s} \ln \left(\frac{R_{s}-R_{0}}{R_{s}-R}\right)\right]
$$

The NW shape is thus controlled by three parameters, the $\beta$ dependent effective $\mathrm{V} / \mathrm{III}$ influx ratio $F_{53}$, the shape constant $\varepsilon$, and the stable radius $R_{s}$, defined as follows

$$
\begin{gathered}
F_{53}=\frac{I_{5}[1+a(\beta)]}{I_{3}}, \quad \varepsilon=\frac{\Omega_{3}}{\Omega_{35} f(\beta)}, \\
R_{s}=\frac{2 \Lambda_{3}}{\pi \varphi(\beta)} \frac{1}{\left(F_{53}-1\right)}
\end{gathered}
$$

The stable radius exists only when $F_{53}>1$, as in ref 22 . Otherwise, the radius always grows with time or with the NW length as in refs 21 and 54 .

According to the expressions given in ref 51, $\varphi\left(\beta_{\max }\right)=1 /$ $\sin ^{2} \beta_{\max }=1.70$ for $\beta_{\max }=130^{\circ}$ and $\alpha=45^{\circ}$, decreasing to 
$\varphi\left(\beta_{\min }\right)=0.854$ for $\beta_{\min }=90^{\circ}$. The $\varepsilon$ values equal $\varepsilon\left(\beta_{\max }\right)=$ 0.0545 and $\varepsilon\left(\beta_{\min }\right)=0.221$ for arsenic-poor and arsenic-rich conditions, respectively. Figure 5 shows the representative

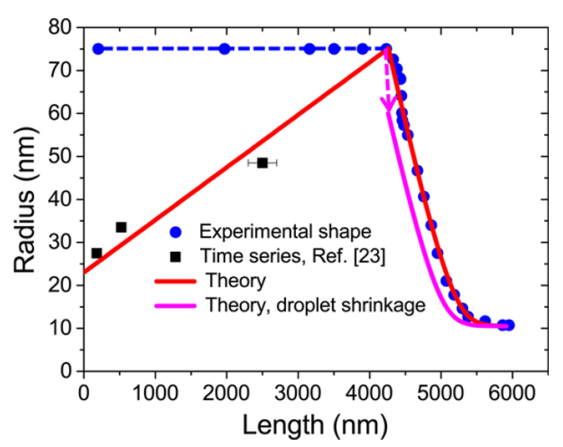

Figure 5. Experimentally measured NN-on-NW shape (symbols) fitted by the model equations (lines). Red line shows the droplet base radius versus length in the case without shrinking the droplet on top of the NW stem, magenta line gives the shape when the droplet instantaneously shrinks from 75 to $60 \mathrm{~nm}$. Transition from tapered to straight geometry of the stem is due to the radial growth by step flow. The experimental values of the radius of NW stem as a function of length for the standard NW growth phase are taken from the data points of ref 26 (see SI3.).

shape of the NW obtained from the TEM image given in Figure 3a, with a $4250 \mathrm{~nm}$ long stem of a constant radius of 75 $\mathrm{nm}$, followed by a tapered section which is transitioned to the top NN with a constant radius of $10 \mathrm{~nm}$ after $\sim 5250 \mathrm{~nm}$ length. The best fit for the shape of the tapered section is obtained from eq 6 at the known $R_{s}=10 \mathrm{~nm}$ and initial conditions with $F_{53}=1.63$. This $F_{53}$ is larger than unity, explaining why the stable NN radius exists. From eq 7 for $R_{s}$, we obtain a very short diffusion length of gallium on the NW side facets of only $8.4 \mathrm{~nm}$. This small value may look surprising at first glance, compared with the previously reported $\Lambda_{3}$ on the order of several hundreds of nanometers (see ref 14 for a review). However, in ref 21, the diffusion length of gallium in the regime of radial growth of gallium-assisted GaAs NWs was found negligible, and this was explained by a high density of surface steps on the sidewalls. Similar explanation looks even more reasonable for highly tapered NN sections where we apply eqs 6 and 7. Indeed, if we assume monatomic steps, the observed $65 \mathrm{~nm}$ decrease of the radius along $\sim 1200 \mathrm{~nm}$ length would require $6 \mathrm{~nm}$ long steps, which is close to the estimate for the diffusion length obtained from the shape fit.

As for the VLS growth of the NW stem, it should proceed under gallium-rich conditions without any stable radius. According to the data of ref 26, the droplet radius scales almost linearly with time after an incubation stage. Assuming linear radius-length dependence in the standard growth mode, as in ref 21, we neglect the logarithmic term in eq 6 and fit the linear shape between the initial radius of $23 \mathrm{~nm}$ (the data points of ref 26) and the measured NW radius after $60 \mathrm{~min}$ of growth $(75 \mathrm{~nm})$, as shown in Figure 5 . This yields $F_{53}=0.82$ for NWs, while it equals 0.59 from the $\mathrm{As}_{4} \mathrm{BEP}$ ratio of 2.75 and $F_{53}=1.63$ in the thinning stage. This discrepancy is most probably explained by different fractions of re-emitted arsenic collected by the droplets seated on top of NNs and NWs, with the collection coefficient $a$ being slightly lower for smaller contact angle droplets. In any case, the $F_{53}$ value is smaller than unity for the standard NWs, which explains why their radius never stabilizes.
In conclusion, we have demonstrated the radius modulation of self-assisted GaAs NWs in the form of NNs with the stable radius down to $10 \mathrm{~nm}$ or even less. The two growth regimes originate from the existence of the two stable contact angles which stabilize the droplet shape with an increasing or constant radius. The findings are validated both by experiments and a theoretical model, which is able to predict the NW-to-NN shape evolution quite accurately. The refinement of the growth theory beyond the standard assumption of a single stable geometry of the growth interface should apply to a wide range of VLS NWs. The demonstrated structures can be very useful in applications with a requirement for strong lateral confinement of carriers, such as quantum light sources and detectors. We also believe that this relatively simple growth strategy can be used in other material systems including self-assisted GaP and InAs NWs and further extended to gold-catalyzed VLS growth.

Methods. Thermal oxide is used as a mask to define the arrays of holes on four inch $<111>$ P-doped Silicon wafers with a resistivity of $0.1-0.5 \Omega \mathrm{cm}$. Substrates are coated with ZEP$520 \mathrm{~A}$ resist at $8000 \mathrm{rpm}$ for $1 \mathrm{~min}$ followed by baking at 200 ${ }^{\circ} \mathrm{C}$ for $5 \mathrm{~min}$. This process yields a nominal resist thickness of approximately $100 \mathrm{~nm}$. A VISTEC EBPG 5000+ E-beam tool operating at $100 \mathrm{kV}$ was used to define the pattern. The pattern consisted of a square arrangement of holes of sizes ranging between 30 and $90 \mathrm{~nm}$; the interhole distance or pitch was varied between 200 and $2000 \mathrm{~nm}$ on the same substrate. The pattern is transferred on the oxide layer by a combination of wet-chemical etching based on 7:1 buffered hydrofluoric acid solution (BHF) and reactive ion etching (RIE) using SPTS APS (dielectric etcher) with $\mathrm{CHF}_{3} / \mathrm{SF}_{6}$ chemistry. After patterning, the wafers were diced into $35 \times 35 \mathrm{~mm}^{2}$ square chips sized for the MBE sample holder. To ensure an oxidefree surface in the holes, the chips were shortly dipped in the $\mathrm{BHF}$ solution prior to the introduction in the UHV chamber. All NWs growth took place in DCA P600 MBE machine. The final oxide thickness before loading to machine was $10 \pm 1 \mathrm{~nm}$ for all substrate. The prepared chips were subsequently annealed at $500{ }^{\circ} \mathrm{C}$ for $2 \mathrm{~h}$ in UHV to ensure a pristine surface free of water and organic molecules. The samples were then transferred to the growth chamber. There, they were degassed at $770{ }^{\circ} \mathrm{C}$ for $30 \mathrm{~min}$ to further remove any possible surface contaminants.

In the first step, Ga was predeposited for $10 \mathrm{~min}$ at beam equivalent pressure (BEP) of Ga of $1.4 \times 10^{-7}$ Torr (corresponding to a nominal Ga growth rate of $1.1 \AA / \mathrm{s}$ ) by keeping the shutter opened. Once growth temperature had been reached, $\mathrm{As}_{4}$ source was opened as following for $60 \mathrm{~min}$ at beam equivalent pressure (BEP) of $\mathrm{As}_{4}$ of $2 \times 10^{-6} \mathrm{Torr}$, at a substrate temperature of $634^{\circ} \mathrm{C}$ measured by pyrometer, and with $7 \mathrm{rpm}$ rotation.

The morphological and structural properties of GaAs NWs were characterized by using a Zeiss MERLIN SEM microscope and by using an FEI Tecnai OSIRIS TEM microscope operated at $200 \mathrm{kV}$ in the conventional and HRTEM modes. The microscope has a point resolution of $0.24 \mathrm{~nm}$.

We acquired the Raman spectra by means of a commercial micro-Raman Renishaw spectrometer. The laser (power density about $2.6 \mathrm{~kW} / \mathrm{cm}^{2}$ ) was sent to the sample by a $100 \times$ objective and the scattered light was collected through the same objective and sent to a diffraction grating with 1800 $l / \mathrm{mm}$. The Raman signal was detected by a charge-coupled 
device (CCD) after suppression of the Rayleigh signal by a notch filter.

\section{ASSOCIATED CONTENT}

\section{S Supporting Information}

The Supporting Information is available free of charge on the ACS Publications website at DOI: 10.1021/acs.nanolett.7b03126.

Analysis of contact angle, further TEM analysis of other nanowires and nanoneedles, data concerning the evolution of radius of nanowire upon nanoneedle formation, scanning electron micrograph of a large arrays, additional information on Raman spectroscopy on nanowires and nanoneedles (PDF)

\section{AUTHOR INFORMATION}

\section{Corresponding Author}

*E-mail: anna.fontcuberta-morral@epfl.ch.

\section{ORCID}

Vladimir G. Dubrovskii: 0000-0003-2088-7158

Anna Fontcuberta i Morral: 0000-0002-5070-2196

\section{Author Contributions}

W.K. and V.G.D. contributed equally to this work.

Notes

The authors declare no competing financial interest.

\section{ACKNOWLEDGMENTS}

V.G.D. thanks the Ministry of Education and Science of the Russian Federation for financial support under Grant No. 14.587.21.0040 (project ID RFMEFI58717X0040). EPFL coauthors thank funding through SNF by ERAnet-Russia Grant No. IZLRZ2 163861, by the NCCR QSIT and Project No. 200021_169908 and the H2020 program through the project INDEEDD.

\section{REFERENCES}

(1) Grundmann, M. The Physics of Semiconductors; Springer, 2006.

(2) Yoffe, A. D. Adv. Phys. 1993, 42 (2), 173-262.

(3) Reimer, M. E.; Bulgarini, G.; Akopian, N.; Hocevar, M.; Bavinck, M. B.; Verheijen, M. A.; Bakkers, E. P. A. M.; Kouwenhoven, L. P.; Zwiller, V. Nat. Commun. 2012, 3, 737.

(4) Muskens, O. L.; Rivas, J. G.; Algra, R. E.; Bakkers, E. P. A. M.; Lagendijk, A. Nano Lett. 2008, 8 (9), 2638-2642.

(5) Krogstrup, P.; Jørgensen, H. I.; Heiss, M.; Demichel, O.; Holm, J. V.; Aagesen, M.; Nygard, J.; Fontcuberta i Morral, A. Nat. Photonics 2013, 7 (4), 306-310.

(6) Heiss, M.; Russo-Averchi, E.; Dalmau-Mallorquí, a.; Tütüncüoğlu, G.; Matteini, F.; Rüffer, D.; Conesa-Boj, S.; Demichel, O.; Alarcon-Lladó, E.; Fontcuberta i Morral, a. Nanotechnology 2014, 25 (1), 014015.

(7) Frederiksen, R. S.; Alarcon-Llado, E.; Krogstrup, P.; Bojarskaite, L.; Buch-Månson, N.; Bolinsson, J.; Nygård, J.; Fontcuberta i Morral, A.; Martinez, K. L. ACS Photonics 2016, 3 (7), 1208-1216.

(8) Frederiksen, R. S.; Alarcon-Llado, E.; Madsen, M. H.; Rostgaard, K. R.; Krogstrup, P.; Vosch, T.; Nygård, J.; Fontcuberta I Morral, A.; Martinez, K. L. Nano Lett. 2015, 15 (1), 176-181.

(9) Qing, Q.; Jiang, Z.; Xu, L.; Gao, R.; Mai, L.; Lieber, C. M. Nat. Nanotechnol. 2013, 9 (2), 142-147.

(10) Duan, X.; Gao, R.; Xie, P.; Cohen-Karni, T.; Qing, Q.; Choe, H. S.; Tian, B.; Jiang, X.; Lieber, C. M. Nat. Nanotechnol. 2011, 7 (3), 174-179.

(11) Fountaine, K. T.; Cheng, W. H.; Bukowsky, C. R.; Atwater, H. A. ACS Photonics 2016, 3 (10), 1826-1832.
(12) Björk, M. T.; Thelander, C.; Hansen, A. E.; Jensen, L. E.; Larsson, M. W.; Wallenberg, L. R.; Samuelson, L. Nano Lett. 2004, 4 (9), 1621-1625.

(13) Kammhuber, J.; Cassidy, M. C.; Zhang, H.; Gul, O.; Pei, F.; De Moor, M. W. A.; Nijholt, B.; Watanabe, K.; Taniguchi, T.; Car, D.; Plissard, S. R.; Bakkers, E. P. A. M.; Kouwenhoven, L. P. Nano Lett. 2016, 16 (6), 3482-3486.

(14) Dubrovskii, V. G. Theory of VLS Growth of Compound Semiconductors, in Semiconductors and Semimetals, Fontcuberta i Morral, A.; Dayeh, S. A.; Jagadish, C., Eds., vol. 93, Academic Press: Burlington, 2015; pp 1-78.

(15) Gil, E.; Dubrovskii, V. G.; Avit, G.; André, Y.; Leroux, C.; Lekhal, K.; Grecenkov, J.; Trassoudaine, A.; Castelluci, D.; Monier, G.; Ramdani, R. M.; Robert-Goumet, C.; Bideux, L.; Harmand, J. C.; Glas, F. Nano Lett. 2014, 14 (7), 3938-3944.

(16) Dong, Z.; André, Y.; Dubrovskii, V. G.; Bougerol, C.; Leroux, C.; Ramdani, M. R.; Monier, G.; Trassoudaine, A.; Castelluci, D.; Gil, E. Nanotechnology 2017, 28 (12), 125601.

(17) Moewe, M.; Chuang, L. C.; Crankshaw, S.; Chase, C.; ChangHasnain, C. Appl. Phys. Lett. 2008, 93 (2), 023116.

(18) Plissard, S.; Larrieu, G.; Wallart, X.; Caroff, P. Nanotechnology 2011, 22, 275602.

(19) Munshi, A. M.; Dheeraj, D. L.; Fauske, V. T.; Kim, D.-C.; Huh, J.; Reinertsen, J. F.; Ahtapodov, L.; Lee, K.; Heidari, B.; van Helvoort, A.; Fimland, B.-O.; Weman, H. Nano Lett. 2014, 14 (2), 960-966.

(20) Russo-Averchi, E.; Vukajlovic Plestina, J.; Tütüncüoglu, G.; Matteini, F.; Dalmau-Mallorquí, A.; De La Mata, M.; Rüffer, D.; Potts, H. A.; Arbiol, J.; Conesa-Boj, S.; Fontcuberta i Morral, A. Nano Lett. 2015, 15 (5), 2869-2874.

(21) Priante, G.; Ambrosini, S.; Dubrovskii, V. G.; Franciosi, A.; Rubini, S. Cryst. Growth Des. 2013, 13 (9), 3976-3984.

(22) Dubrovskii, V. G.; Xu, T.; Álvarez, A. D.; Plissard, S. R.; Caroff, P.; Glas, F.; Grandidier, B. Nano Lett. 2015, 15 (8), 5580-5584.

(23) Tersoff, J. Nano Lett. 2015, 15 (10), 6609-6613.

(24) Somaschini, C.; Bietti, S.; Trampert, A.; Jahn, U.; Hauswald, C.; Riechert, H.; Sanguinetti, S.; Geelhaar, L. Nano Lett. 2013, 13, 36073613.

(25) Krogstrup, P.; Hannibal Madsen, M.; Hu, W.; Kozu, M.; Nakata, Y.; Nygård, J.; Takahasi, M.; Feidenhans'l, R. Appl. Phys. Lett. 2012, 100, 093103.

(26) Vukajlovic-Plestina, J.; Kim, W.; Dubrovskii, V. G.; Tütüncüoğlu, G.; Lagier, M.; Potts, H.; Friedl, M.; Fontcuberta i Morral, A. Nano Lett. 2017, 17, 4101.

(27) Loitsch, B.; Rudolph, D.; Morkötter, S.; Döblinger, M.; Grimaldi, G.; Hanschke, L.; Matich, S.; Parzinger, E.; Wurstbauer, U.; Abstreiter, G.; Finley, J. J.; Koblmüller, G. Adv. Mater. 2015, 27 (13), 2195-2202.

(28) Bauer, B.; Rudolph, A.; Soda, M.; Fontcuberta i Morral, A.; Zweck, J.; Schuh, D.; Reiger, E. Nanotechnology 2010, 21 (43), 435601.

(29) Colombo, C.; Spirkoska, D.; Frimmer, M.; Abstreiter, G.; Fontcuberta I Morral, A. Phys. Rev. B: Condens. Matter Mater. Phys. 2008, 77 (15), 2-6.

(30) Glas, F.; Ramdani, M. R.; Patriarche, G.; Harmand, J. C. Phys. Rev. B: Condens. Matter Mater. Phys. 2013, 88 (19), 1-14.

(31) Waugh, J. L. T.; Dolling, G. Phys. Rev. 1963, 132 (6), 24102412.

(32) Kiefer, W.; Mazzolini, A. P.; Stoddart, P. R. J. J. Raman Spectrosc. 2007, 38 (12), 1538-1553.

(33) Sood, A. K.; Menéndez, J.; Cardona, M.; Ploog, K. Phys. Rev. Lett. 1985, 54 (19), 2111-2114.

(34) Ketterer, B.; Arbiol, J.; Fontcubertai Morral, A. Phys. Rev. B: Condens. Matter Mater. Phys. 2011, 83 (24), 1-6.

(35) Swinkels, M. Y.; van Delft, M. R.; Oliveira, D. S.; Cavalli, A.; Zardo, I.; van der Heijden, R. W.; Bakkers, E. P. A. M. Nanotechnology 2015, 26 (38), 385401.

(36) Jacobsson, D.; Panciera, F.; Tersoff, J.; Reuter, M. C.; Lehmann, S.; Hofmann, S.; Dick, K. A.; Ross, F. M. Nature 2016, 531 (7594), 317-322. 
(37) Dheeraj, D. L.; Munshi, a M.; Scheffler, M.; van Helvoort, a T. J.; Weman, H.; Fimland, B. O. Nanotechnology 2013, 24, 15601.

(38) Cirlin, G. E.; Dubrovskii, V. G.; Samsonenko, Y. B.; Bouravleuv, A. D.; Durose, K.; Proskuryakov, Y. Y.; Mendes, B.; Bowen, L.; Kaliteevski, M. A.; Abram, R. A.; Zeze, D. Phys. Rev. B: Condens. Matter Mater. Phys. 2010, 82 (3), 035302.

(39) Dubrovskii, V. G.; Cirlin, G. E.; Sibirev, N. V.; Jabeen, F.; Harmand, J. C.; Werner, P. Nano Lett. 2011, 11 (3), 1247-1253.

(40) Plissard, S.; Dick, K. a; Larrieu, G.; Godey, S.; Addad, A.; Wallart, X.; Caroff, P. Nanotechnology 2010, 21 (38), 385602.

(41) Glas, F.; Harmand, J. C.; Patriarche, G. Phys. Rev. Lett. 2007, 99 (14), 3-6.

(42) Dubrovskii, V. G. Cryst. Growth Des. 2017, 17 (5), 2544-2548. (43) Dubrovskii, V. G.; Sibirev, N. V.; Cirlin, G. E. Tech. Phys. Lett. 2004, 30 (8), 682-686.

(44) Kashchiev, D. Cryst. Growth Des. 2006, 6 (5), 1154-1156.

(45) Nebol'sin, V. A.; Shchetinin, A. A. Inorg. Mater. 2003, 39 (9), 899-903.

(46) Wen, C. Y.; Tersoff, J.; Hillerich, K.; Reuter, M. C.; Park, J. H.; Kodambaka, S.; Stach, E. A.; Ross, F. M. Phys. Rev. Lett. 2011, 107 (2), 1-4.

(47) Rosini, M.; Magri, R. ACS Nano 2010, 4 (10), 6021-6031.

(48) Sibirev, N. V.; Timofeeva, M. a.; Bol'shakov, a. D.; Nazarenko, M. V.; Dubrovskiri, V. G. Phys. Solid State 2010, 52 (7), 1531-1538. (49) Gibson, S.; Lapierre, R. Phys. Status Solidi RRL 2013, 7 (10), 845-849.

(50) Ramdani, M. R.; Harmand, J. C.; Glas, F.; Patriarche, G.; Travers, L. Cryst. Growth Des. 2013, 13 (1), 91-96.

(51) Glas, F. Phys. Status Solidi B 2010, 247 (2), 254-258.

(52) Dubrovskii, V. G. J. Cryst. Growth 2016, 440, 62-68.

(53) Dubrovskii, V. G. Appl. Phys. Lett. 2014, 104, 053110.

(54) Gibson, S. J.; Boulanger, J. P.; LaPierre, R. R. Semicond. Sci. Technol. 2013, 28 (10), 105025. 